\title{
Silicon Detector Upgrades for the Tevatron Run 2
}

\author{
M. C. Kruse ${ }^{a *}$

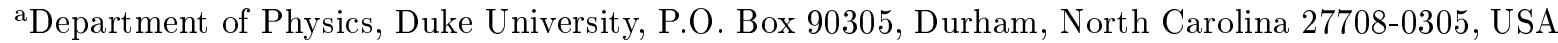

The current silicon devices being used by the DØ and CDF collaborations for the Tevatron Run 2a, which is expected to end in 2005 after accumulating about $2 \mathrm{fb}^{-1}$ of data, will need to be replaced due to radiation damage for the following data collection period designated as Run $2 \mathrm{~b}$. We will discuss these silicon replacement plans, the more uniform design of the detectors between $D \varnothing$ and CDF, and the current status of their fabrication.

\section{INTRODUCTION}

The goal of the Tevatron Run $2 \mathrm{a}$ is to deliver about $2 \mathrm{fb}^{-1}$ of integrated luminosity to the $\mathrm{D} \varnothing$ and CDF detectors by 2005 [1]. Based on some recent improvements to the Tevatron performance, we expect to accumulate about $100 \mathrm{pb}^{-1}$ by the end of 2002, and $400 \mathrm{pb}^{-1}$ by the end of 2003 . The radiation damage that will be incurred by the Run 2a DØ and CDF silicon detectors, both to the bulk silicon and the frontend readout electronics, will necessitate at least the partial replacement of these systems.

Following Run 2a, and after a short shutdown period, Tevatron running will resume as Run $2 \mathrm{~b}$, with the ultimate integrated luminosity goal of $15 \mathrm{fb}^{-1}$ per experiment. Such an integrated luminosity is essential for the sensitivity to the discovery of a light Higgs boson at the Tevatron. The Higgs sector is the last cornerstone of the Standard Model yet to be directly explored, and our findings, or lack thereof, will have deep ramifications to our understanding of matter. A light Higgs boson $\left(M_{H}<140 \mathrm{GeV} / c^{2}\right)$ decays predominantly to a $b \bar{b}$ pair, therefore, critical to the discovery of a light Higgs is the detection of the secondary vertices from $b$-quark decays, accomplished with high efficiency through the use of silicon vertex detectors. In Run 2 we expect to be able to "tag" at least one $b$-quark jet from a Higgs decay with about $60 \%$ efficiency.

The silicon vertex detectors have been designed for both the CDF and DØ Run 2b upgrade projects, with construction and prototyping now

\footnotetext{
${ }^{*}$ Representing the CDF and $\mathrm{D} \varnothing$ collaborations
}

well underway. The main factors governing the design of these detectors were the following.

- Greater radiation tolerance: both sensors and frontend electronics have been designed to operate for at least $15 \mathrm{fb}^{-1}$.

- Simplicity of construction to meet the scheduled completion in 2005/2006.

- Retention or improvement of the tracking capabilities of the Run $2 \mathrm{a}$ silicon systems.

- Preservation of as much of the existing infrastructure as possible. This includes most of the DAQ systems, the external cooling systems, and the available space and structural support, present in both the CDF and D $\varnothing$ experiments.

- Given the infrastructure differences between the two experiments, exploit the areas where we can benefit from common design features. This includes common readout chips, and sensor specifications.

\section{MECHANICAL LAYOUTS}

Both the DØ and CDF Run 2b silicon detectors have a 6 -layer geometry arranged in 2 barrels, each about $60 \mathrm{~cm}$ long, joined at $z=0$. The Run 2a DØ silicon tracker [2] will be replaced in its entirety. The outer layers of the CDF silicon system, the ISL [3], will remain unchanged, with the present SVX 2a and Layer 00 devices [4] being replaced. The geometrical similarity is apparent in Figure 1, which shows the cross-sectional views of the replacement detectors.

The 6 layers for both detectors (labelled 0 to 5) vary in radii from about $2 \mathrm{~cm}$ to $16 \mathrm{~cm}$. This 


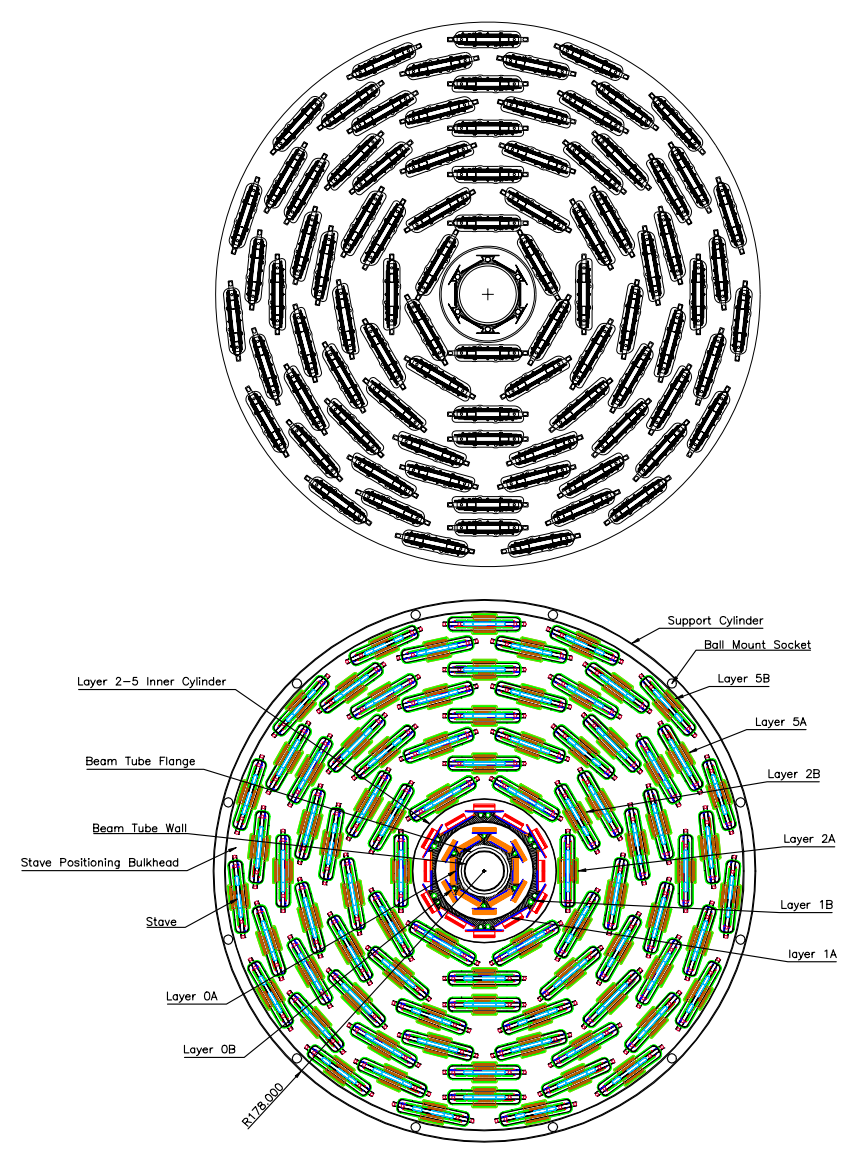

Figure 1. Transverse views of the mechanical layout of the CDF(top) and DØ (bottom) silicon detector upgrades.

fills more of the tracking volume with silicon sensors as opposed to the Run 2a designs, as a lot of the readout electronics have been pushed out to the ends of the barrels. The basic detector unit is the "stave". Details in the stave design differ between $\mathrm{D} \varnothing$ and CDF but the more general design features are similar, in that they are composed of two layers of single-sided silicon sensors upon which the SVX4 readout chips are mounted (by way of "hybrid" circuit boards), and between which the cooling channels have been integrated. A schematic of the CDF stave is shown

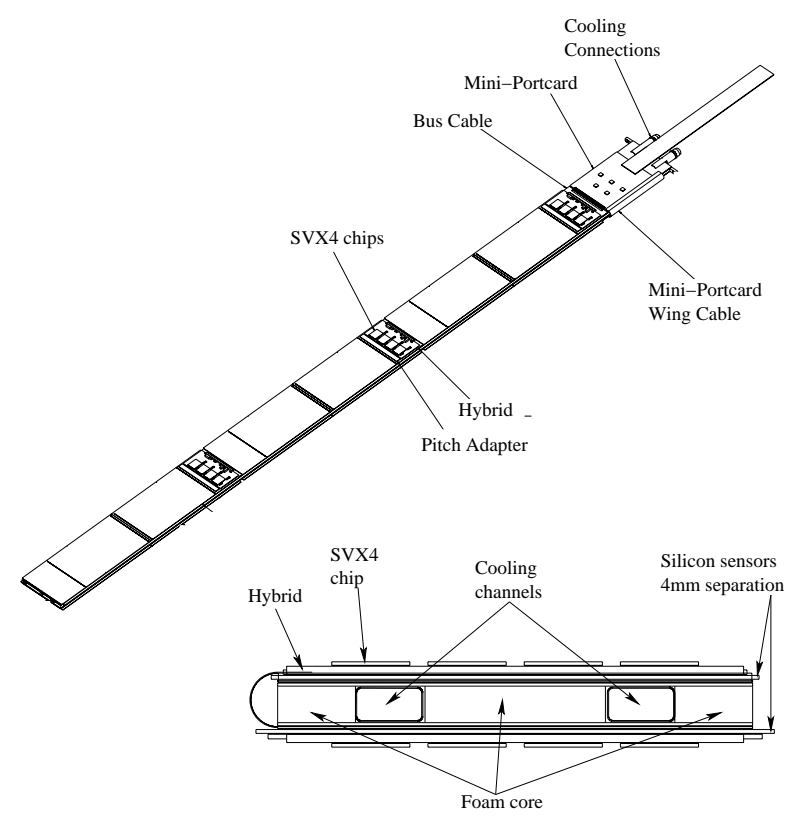

Figure 2. Isometric (top) and cross-sectional (bottom) views of the CDF stave design for all the outer layers.

in Figure 2. There will be no $90^{\circ}$ stereo sensors, however, layers 2 through 5 have either $2.5^{\circ}$ or $1.2^{\circ}$ small-angle-stereo sensors on one side of the staves. DØ will have the same stave design for layers 2 to 5 , and CDF for layers 1 to 5 . Therefore, both detectors will be entirely comprised of only 3 sensor types (axial and stereo for the outer layers, and axial for the inner layer(s)), greatly reducing the design complexity. The sensor pitches differ slightly for both experiments: DØ use a strip(readout) pitch of $30 \mu m(60 \mu \mathrm{m})$ for the outer layers (2 to 5$), 29 \mu \mathrm{m}(58 \mu \mathrm{m})$ for layer 1 , and $25 \mu \mathrm{m}(50 \mu \mathrm{m})$ for layer 0 ; and $\mathrm{CDF}$ uses pitches of $37.5 \mu \mathrm{m}(75 \mu \mathrm{m})$ for the outer layers (1 to 5 ), and $25 \mu \mathrm{m}(50 \mu \mathrm{m})$ for layer 0 . The smaller pitch used for the DØ outer layers, means 5 SVX4 chips are required to readout the channels spanning the width of a sensor, as opposed to 4 readout chips for CDF.

The single-sided sensors can be operated at higher bias voltages than the double-sided Run 
2a sensors which are limited to about $100 \mathrm{~V}$ by the coupling of the applied voltages to each side. As the bulk silicon suffers radiation damage the voltages required to maintain full depletion increase, giving the single-sided sensors a considerably longer lifetime in high radiation environments. It is envisioned by the end of Run $2 \mathrm{~b}$ bias voltages for the inner layers will need to be about $600 \mathrm{~V}$. The use of single-sided sensors also increases the simplicity of the construction. Initial tests on prototype sensors have been very encouraging, with measured leakage currents being about $0.15 \mu \mathrm{A}$ all the way to $1000 \mathrm{~V}$ (the specification being $0.20 \mu \mathrm{A}$ ). Construction of prototype staves is now underway.

\section{DATA ACQUISITION}

The frontend readout system has undergone the most significant changes from the Run 2a DAQ. The rest of DAQ system has largely remained intact.

Both CDF and DØ will use the SVX4 readout chip, replacing the current SVX3 and SVX2 chips respectively. The SVX4 chip is based on the SVX3 design with an analog front-end, digital back-end, a 42 cell pipeline, and 128 channels wide, but produced with 0.25 micron technology, giving it an intrinsic radiation hardness that is expected to withstand the total doses to the inner layers. The sub-micron technology has also resulted in the halving of the operating voltage to $2.5 \mathrm{~V}$. Prototype SVX4 chips were received in June 2002 and are fully functional with no major fixes required. Further studies, including noise tests are ongoing.

The SVX4 chips are mounted on, and serviced by, hybrid circuit boards, which are in turn mounted on the staves. The same substrate and etching technology for the hybrids are used by both DØ and CDF, however, the hybrid designs are somewhat different. The hybrids use a $\mathrm{BeO}$ substrate, and are smaller than in Run 2a, significantly reducing their impact in the tracking volume. The one exception is the inner CDF layer, which uses the same hybrid design as for the current CDF Layer 00 detector, with alumina substrate and placed outside of the tracking volume.
CDF has two hybrid types, one with 2 SVX4 chips for layer 0 , and a 4-chip version for the outer layers. As can be seen in Figure 2 each hybrid reads out a 2-sensor module. A total of 72 2-chip and 1080 4-chip hybrids are required.

DØ has four hybrid types and utilise a doubleended design which reduces the total hybrid readout count. Outer layers (2 to 5) are read out by 10-chip hybrids ( 5 chips on each end), layer 1 by double-ended 6-chip hybrids, and layer 0 by single-ended 2-chip hybrids. A total of 672 10chip, 72 6-chip, and 144 2-chip hybrids are required.

Circuit boards outside the tracking volume take signals from the hybrids and feed them to the rest of the DAQ system, similarly to the Run 2a system, however, with some of the hybrid and portcard functionality moved further from the tracking volume, and with no optical components.

\section{CONCLUSIONS}

Extending the Tevatron Run 2 to $15 \mathrm{fb}^{-1}$ opens up many exciting physics opportunities. The greater integrated luminosity also extends the radiation dose to the existing inner layers of the $\mathrm{CDF}$ and DØ silicon vertex detectors beyond their design limits, which has compelled the design and construction of silicon detector upgrades. Both the DØ and CDF upgrades have many similarities within the constraints of their existing infrastructure, the most notable being similar sensor specifications, and the use of the same SVX4 readout chip. Progress on many components has been excellent, for example, we have SVX4 chips working on hybrids, and working sensors have been delivered to both experiments. These devices will play a crucial role in extending our capabilities for some very important physics goals this decade.

\section{REFERENCES}

1. K. Gounder, these proceedings.

2. G. Ginther, these proceedings.

3. A. Affolder, et al. (CDF collaboration), Nucl. Instrum. Meth. A461:216-218,2001

4. S. Nahn, these proceedings. 